\title{
A Personalized Drug Delivery System for the Treatment of Cardiac Arrhythmias
}

\author{
Bhakti Ganesh Dalvi, Kajal Khushal Darje \\ M Pharm, Department of Pharmaceutics, PES'S Modern College of Pharmacy, Nigdi, Pune, India
}

\begin{abstract}
Now a days many people are suffering from cardiovascular diseases, it gets major challenge in front of pharmaceutical and medical science to treat cardiovascular diseases. Amongst different cardiovascular diseases arrhythmia is the most emerging disease in the world especially in developed countries, it is estimated that arrhythmic syndrome are responsible for death of 50\% of population i.e. death rate is increasing day by day so it is essential to develop something new to treat cardiac arrhythmia in safe and effective way. Personalized drug delivery system may help to tackle many problems arising with antiarrhythmic drugs as they give same treatment to every individual rather studying his or her genetic makeup so it shows some negative effects on human body. Personalized therapy includes the detailed study of patients genetic makeup and other factors such as age, habits, medical history, hereditary diseases, lifestyle, diet, physiological condition, disease state and economical condition. This treatment is identical to each individual means it may vary person to person hence it is called as personalized or customized drug delivery system.
\end{abstract}

Keywords: personalized medicine, cardiovascular diseases, genetic architecture arrhythmia, genetic variants

\section{Introduction: $:^{[1,2,3]}$}

Personalized medicine is the form of medicine that uses information about a person's genes, proteins, and environment to prevent, diagnose and treat disease. Personalized medicine or precision medicine is a medical model that seperates people into different groups- with medical decision, practices, interventions and products ${ }^{[1]}$. Personalized medicine involves:

1) Making the treatment as individualized as disease.

2) To predict an individual's susceptibility to disease.

3) Young but rapidly advancing field.

4) Use of new method of molecular analysis. ${ }^{[2]}$

The goal of personalized medicine is to tailor diagnostics and treatments to our individual genetic makeup, which will increase the rate of success in curing patients with the right drug of the right dose at right time.

Genetics realize that before they can personalized diagnostic tests and cures, they have to decode the complex machineries behind our genes and their variants. To do so they need a lot of data not only genetics, biological and clinical but also lifestyle information about lot of individuals from diverse background.

Development of concept of Personalized medicine:

a) In personalized medicine, diagnostic testing is often employed for selecting appropriate and optimal therapies based on the context of patient's genetic content.

b) The use of genetic information has played a major role in certain aspects of personalized medicine (e.g. Pharmacogenomics and Pharmacogenetics)

c) Every person has a unique variation of the human genome that's why person may show different reaction for same medicine ${ }^{[3]}$.

\subsection{Precision health}

a) A person genetic makeup at or before birth and determination of their unique disease risk done. b) Information helps in the customization of the healthmonitoring devices that a person can use in his day to day life.

c) There are some devices which can measure environmental and physiological parameters and transmit data to the integrated health portal.

d) The portal can co-ordinate with health decision-making by analyzing data to suggest an actionable response to the appropriate type of health care provider.

e) Simple, low-risk interference, such as diet or weight change, can be presented directly to the patient along with education and lifestyle guidance.

f) More complex disease and therapy management involves the physician, hospital, and outpatient centers. Customized monitoring and intervals of surveillance can be adjusted appropriately on a continual basis.

g) The main goal personalized medicine is to engage patient to maintain their health to avoid many disease and return to hospital.

\subsection{Arrhythmia- An emerging cardiovascular disease}

Cardiac arrhythmia is type of cardiovascular disease increasing with higher risk day by day. It is responsible for cardiovascular mortality and morbidity.

Definition: A condition in which the heart beats with an irregular or abnormal rhythm. ${ }^{[4]}$

Atrial fibrillation (AF) is the most common cardiac arrhythmia in developed contries. AF is associated with increase mortality and morbidity due to thromboembolism, stroke and worsening of pre-existing heart failure. AF is an irregular and often rapid heart rate that can increase risk of stroke and heart failure. ${ }^{[5]}$

Symptoms:

1) Palpilation

2) Confusion

3) Dizziness, light headache.

4) Fainting, fatigue. 
5) Loss of ability to exercise.

6) Shortness of breath.

Factors responsible for arrhythmia:

1) Physiological factors or diseased condition:

- Electrolyte imbalance

- Diabetic condition

- Heart disease like congestive heart failure.

- Hypertension

- Hyperthyroidism

2) Lifestyle of person:

- Alcohol abuse

- Drug abuse

- Excessive coffee consumption

- Mental stress

- Smoking

3) Other medical treatment:

- Some dietary supplement.

- Some herbal supplement. ${ }^{[6]}$

\subsection{Familial Arrhythmic Syndrome}

Following are some different types of arrhythmia:

a) Extrasystole (ES): These are premature ectopic beats due to abnormal automaticity of after depolarization arising from an ectopic focus in the atrium (AES), A-V node (nodal ES) or ventricles (VES). The QRS complex in VES is broder and abnormal in shape.

b) Paroxysmal supraventricular tachycardia (PSVT): It is sudden onset episode of atrial tachycardia (rate 150$200 / \mathrm{min}$ ) with 1:1 atrio-ventricular conduction; mostly due to circus movement type of recently occurring within or around the A-V node or using an accessory pathway between atria and ventricles (Wolf Parkinson white syndrome). ${ }^{[7]}$

c) Atrial flutter: atria beat at a rate of 200-350 per min and there is a physiological $2: 1$ to $4: 1$ or higher AV blocks. This is mostly due to stable reentrant circuit in the right atrium but some cases may be due to rapid discharge of an atrial focus.

d) Atrial fibrillation (AF): Atrial fibers are activated asynchronously at a rate of $350-550$ per min (due to elecrophysiological inhomogeneity of atrial fibres), associated with grossly irregular and often fast (100-160 per min) ventricular response. Atria remains dilated and quiver like bags of worms.

e) Ventricular tachycardia (VT): It is run of four or more consecutive ventricular Extrasystole. It may be sustained or nonsustained arrhythmia, and is due either to discharge from ectopic focus. After depolarization or single site (monomorphic) or multiple site (polymorphic) reentry circuit.

f) Torsades de point: It is life threatening form of polymorphic ventricular tachycardia with rapid asynchronous complexes and an undulating baseline on ECG. It is generally associated wit long QT interval.

g) Ventricular fibrillation: It is grossly irregular, rapid and fractionated activation of ventricles resulting in coordinated contraction of its fibres with loss of pumping function. It is fatal unless reverted with in $2-5 \mathrm{~min}$ is the most common cause of sudden cardiac death. h) Atrio-ventricular block: It is due to depression of impulse conduction through the A-V node and bundle of his, mostly due to vagal influence of ischemia. ${ }^{[8]}$

\subsection{Treatment of Arrhythmia:}

a) Vagal maneuvers: Certain movements that patient can stop some type of arrhythmia which start above the lower half of the heart.

b) Medication: Only medications will not cure arrhythmia completely this may help in the reducing episodes of tachycardia and can help with proper electrical conduction of the heart.

c) Cardioversion: In this electrical shocks are used to beat heart in regular rhythm.

d) Ablation therapy: An instrument which is implanted near the left collarbone and detects heart rhythm; if that instrument detects an abnormally fast rhythm, it stimulates the heart to return to a normal rhythm.

e) Maze procedure: A series of surgical incision are made in the heart. They then heal into scars and form blocks. These blocks guide the electrical impulses, helping the heart to beat efficiently.

f) Ventricular aneurysm surgery: Sometimes, an bulge in the blood vessel that leads to the heart causes an arrhythmia. If other treatments do not work, a surgeon may remove the aneurysm (bulge).

g) Coronary bypass surgery: Arteries or veins from anywhere in the patients body are grafted to the coronary arteries to bypass any regions that have become narrow, and improve the blood supply to heart muscle(myocardium). ${ }^{[9]}$

\section{Personalized Medicine and Arrhythmia}

Personalized medicine is a novel medical model with all treatment and practices being specific to individual patients in whatever ways possible. In the era of genomics, personalized medicine combines the genetic information for additional benefits in preventive and therapeutic strategies.

Personalized medicine may allow the physician to provide better therapy for patient in term of efficacy, safety and treatment length to reduce associated costs. The idea of personalizing medicine is increasingly used with the idea of applying information about genomics variations to understand disease risk, progression and variable drug response in an individual.

Response of currently available antiarrhythmic drug is vary from person to person, some patient feels clear cut benefit while other derive no benefits or even develop new arrhythmia. Thus all positive and negative response of antiarrhythmic drugs totally depends upon the persons genetic architecture as each person have unique genetic code. Other than genes some different factors are there which affects the drug response these are environmental factor, person's lifestyle, physiological condition, economical condition, diseased state of person, diet, and family background of person.

The idea of personalized medicine study all the factors responsible for the effect of the antiarrythmic drug along 
with genomic of the person and helps to physician to prescribe proper medicine with better effects. ${ }^{[10]}$

Some antiarrhythmic drug may responsible for the QT prolongation and torsade de points. e.g. drug induced QT prolongation and Torsade de pointes. In this the proposed cellular mechanism of drug-induced prolonged QT interval involves the inhibition of rapid component of the delayed rectifier potassium current. ${ }^{[11]}$ Due to Blocking of potassium channel there is prolongation of the ventricular action potential duration takes place, leading to an excess sodium influx or decrease potassium efflux. This excess of positively charged ions leads to an extended repolarization phase, resulting in a prolonged QT interval and causing arrhythmias such as TdP.

There is an extensive list of medication that can prolong QT intervals in that some antiarrhythmic drugs are class Iadisopyramide, quinidine, procainamide. And class IIIsotalol, dofetilide, ibutilide. ${ }^{[12]}$

\section{Genetic variants and arrhythmia susceptibility}

Monogenic forms of arrhythmia helps to understand the pathophysiology of cardiac arrhythmia in humans. Genotyped families have been systematically identified on a large scale basis, and a variable intra and interfamilial disease expression has been observed.

Genetic and basic electrophysiological investigations have unrevealed a widespread pathophysiological heterogeneity.

Most common diseases are complex genetic traits, with multiple genetic and environmental components, contributing to susceptibility. Common genetic variants, including single nucleotide polymorphism (SNPs) influence susceptibility to common disease or arrhythmia.

The advantage of genetic variants is that the specific variants make some physiologic sense; each variant may make different physiological sense, however this approach is now well recognized to suffer from very frequent failure of replication.

The family approach requires large kindred's. But recent development in genomic sequencing have opened the possibility if identifying new diverse genus even in small families with single affected individuals. ${ }^{[13]}$

\section{Genetic Contributors In Arrhythmia:}

\section{Molecular genetics in arrhythmia:}

Variants of genes open up a variety of ion channels or their respective proteins were found to cause inherited arrhythmias, such as long QT syndrome, brugada syndrome, catecholaminergic polymorphic ventricular tachycardia, progressive cardiac conduction defect, and short QT syndrome.

Functional analysis by using electrophysiological method is precise mechanism underlying their respective pathogenecity. Rapid adoption and spread of genetic test for these disease have offered a unique approach not only for diagnosis, but also for risk assessment and therapeutic decision, and eventually for personalized medicine. ${ }^{[14-18]}$

\section{Modulation Mechanisms Of Heart Rate And Genetic Exacerbation Factors:}

In cardiac conducting system, the sinoatrial node (SAN) is the primary pacemaker component and function as source for automaticity; that is spontaneous depolarization with regular interval.

The SAN displays heterogenous cellular morphology action potential configuration and electrophysiological characteristics. The SAN's major pacemaker site may shift peripherally depending upon various intervational factors:

a) Electrolyte concentration

b) Autonomic nervous stimuli

c) Temperature

The pacemaker tends to shift to the site where electrical activity is least suppressed by extrinsic factors.

The molecular mechanism underlying myocyte firing in the central SAN are characterized by the SAN's unique gene expression profile with minimal expression of $\mathrm{KCNJ} 2$ (inwardly rectifying $\mathrm{K}$ channel) and higher expression of HCN4 (the pacemaker channel).

The absence of KCNJ2 expression allows the resting membrane potential depolarized to enable spontaneous depolarization, while the absence of SCN5A expression can prevent rapid upstroke of action potential. Abundant expression of the HCN4 pacemaker channel promotes spontaneous, slow depolarization is response to phase 4 hyperpolarization.

Due to abundant expression of SCN5A is responsible for the fast upstroke of action potential in phase 0 and because of this there is rapid electrical conduction in the peripheral SAN. Thus loss of function mutation in SCN5A could result in SA exit block, an electrical conduction blockade between the central SAN and surrounding atrial myocytes. ${ }^{[19]}$

\section{Mutation in gene responsible for calcium regulation:}

Gene responsible for sinus bradycardia due to abnormal $\mathrm{Ca}^{2+}$ regulation include the ryanodine receptor RYR2 and the calsequestrin CASQ2, both of which are known to causative genes for catecholaminergic polymorphic ventricular tachycardia (CPVT)

CPVT- related mutations in these genes affect $\mathrm{Ca}^{2+}$ regulations by disrupting its storage and release from sarcoplasmic reticulum during period of exercise, emotional stress resulting in sinus bradycardia and fatal ventricular tachyarrythmia.

The identification of gene mutation contributing to $\mathrm{Ca}^{2+}$ release and storage in the SR served to reinforce the critical role of calcium clocks in maintenance of normal sinus rhythm. ${ }^{[20]}$

Gene involved in cardiac development and bradycardia: Development of the ccs is a complex biological process with the potential to be wrought with problems. Several 
transcription factors, including homeodomain protein and $\mathrm{T}$ box proteins, are essential for ccs morphogenesis and the activation or repression of key regulators genes of the cardiogenic transcription factor genes; GATA4, NK X2-5, TBX3 and TBX5 play key roles in the development of primary and secondary heart fields, while mutations result in congenital heart disease such as patent foramen ovale, itself often related with conduction disorders.

A missense mutation G125R, has been identified in a family suffering from faint digit abnormalities and higher prevalence of atrial fibrillation without heart malformation . atrial fibrillation is believed to be associated with the increased expression of NPPA, GJA5, KCNJ2 and TBX3. ${ }^{[21]}$

\section{Genetic testing and the molecular autopsy:}

In sudden arrhythythmogenic death syndrome, where no cause of death is identified after a comprehensive postmortem examination; genetic testing of the decendent's blood sample collected at post mortem may identified an underlying genetic cause of sudden death. This genetic testing has been termed as molecular autopsy.

DNA extraction from postmortem blood, followed by DNA analysis of selected candidate genes responsible for the main inherited arrhythmogenic disease.

Establishing a definitive cause of death in young SCD case is of major importance, and so the postmortem evaluation is a critical first step in all cases. important aspects of postmortem include the proper and detailed conduct of postmortem examination itself, collection of appropriate sample for analysis (including DNA analysis), careful evaluation of the findings, and a precise and accurate final conclusion are important aspect of the role of the postmortem. This information can thereby provide a therapeutic window for disease and sudden death prevention in at-risk relative. ${ }^{[2]}$

\section{New Genomic Knowledge to Personalized Antiarrhythmic Therapy: \\ Response to currently used antiarrhythmic therapies is vary from person to person, with some patients get clear cut benefit, such as reduction in episodes of paroxysmal atrial fibrillation, while other derive no benefit or even develop new arrhythmias, such LQTS.}

Now genome sequencing, copy number variation, transcriptional readouts, and comprehensive measurement of micro RNA, protein, and metabolic levels provide system approach to probe and predict human health and disease state that has greatly explore the impact of principles of genetics and genomics on clinical medicine. These advances have provided both a conceptual and technological underpinning for the development of the field of genomic medicine as a driver of personalized health care.

\section{Pharmacokinetic variations:}

Pharmacokinetic means body dose to the drug, the determinants of the plasma concentration of antiarrhythmic drugs are important because variation in plasma level is often greater than the desired therapeutic range.
Although considering blood concentration for various subgroups to personalize treatment dose not generate the level of excitement that identifying responders genetically or pathophysiologically does, it is well understood and highly justified example of personalized medicine. The pharmacokinetic of drug is alter when there are changes in the function of organs responsible for absorption, distribution and elimination. The changes in the function of critical organ (eg. Liver kidney) are generally correlated with patient attributes such as age, body weight or comorbidities. Drug development programs now a days investigate on the pharmacokinetic impact of abnormal renal and hepatic function, of sex and age, and of concomitant treatments that could affect metabolism in antiarrhythmic drug therapy, can be attributed to variable drug disposition.

E.g. CYP2D6 is responsible for bio-inactivation of no. of $\beta$ blockers, including metaprolol and timolol and use of these drugs in poor metabolizers can result in high drug concentration and increase risk of bradyaarhythmias and bronchospasm. The sodium channel blocking antiarrhythmic propafenone is also metabolized by CYP2D6. in this case the downstream metabolite 5-hydroxyprofenone retains the sodium channel blocking properties of the parent drug but is devoid of its $\beta$ blocking activity as a results poor metabolizers administer propafenone develop higher concentration of parent drug and display greater heart rate slowing.

These examples highlight the principle that for each drug, a set of gene whose products are responsible for absorption, distribution, metabolism and elimination can be identified and as a consequence, variants in these pathways can be associated with variable drug concentration and thus drug actions.

\section{Pharmacodynamic variation:}

It means drug dose to the body. Most antiarrhythmic agents have a degree of negative ionotropic effect at some concentration and patients with reduce myocardial reserve are especially vuinerable to these effects drugs like tocainide, mexiletin and ecainide appear to cause only minimal myocardial depression. In case of congestive cardiac failuare, some antiarrhythmic drugs have special concern which include diuretic induced hypokalemia, proarrhythmia and some interactions with cardiac glycoside and other drugs, during subsequent outpatient therapy. Patient should take precaution of unexpected reactions, toxicity or electrolyte imbalance.

e.g. calcium channel blocker mibefradil, when used with the simvastatin inhibits CYP3A4 and CYP2D6 which may causes severe muscle damage (rhabdomyolysis) and when used with astemizole, cisapride and terfenadine causes torsade de pointe. ${ }^{[23]}$

\section{Targeting Specific Molecular Mechanism:}

To avoid unexpected reactions it is necessary to understand molecular or cellular basis of arrhythmia or any familial arrhythmic syndrome. Now a days the drugs which are used for the treatment of arrhythmia are developed long before the molecular and cellular basis of arrhythmia was defined but to avoid different side effects or unexpected reaction it is 
essential to study molecular and cellular basis of arrhythmia. $^{[24]}$

\section{Targeting specific molecular mechanism- Atrial fibrillation:}

Atrial fibrillation is the commonest arrhythmia targeted by antiarrhythmic drugs, and the idea, based on the congenital long QT syndrome paradigm, that targeting drugs to specific molecular subtypes of the arrhythmia might improve efficacy is appealing. However, studies delineating such molecular mechanisms are, as outlined above, in their infancy and so it is not yet clinical standard of care to use genetic information to guide therapy in this arrhythmia. One approach is to target mechanisms in distinct functional subsets. For example, some patients develop atrial fibrillation at night or with vagal stimuli, and local application of acetylcholine or analogs to the atrium results in heterogeneous action potential shortening and atrial fibrillation with atrial stimulation. The underlying mechanism is likely shortened atrial action potential duration due to activation of IK-ACh, and compounds targeting this channel are effective prevention of the arrhythmia in experimental animals.

A number of reports have identified a relationship between AF susceptibility alleles at $4 \mathrm{q} 25$ and decrease efficacy of ablation, decrease antiarrhythmic drug therapy efficacy in $\mathrm{AF}$, recurrent arrhythmia after elective cardioversion, and development of atrial fibrillation after cardiac surgery. The mechanisms remain uncertain, but one possibility is that the $4 \mathrm{q} 25$ locus creates an AF substrate that is resistant to these forms of therapy by the time of initial clinical presentation; this could arise, for example, through enhanced atrial fibrosis occurring prior to initial presentation. If this were to be the case, then genetic testing to identify individuals at risk prior to initial presentation would be a possible therapeutic approach. Other studies have identified variants in beta adrenergic receptor genes as modulators of drugs used to slow ventricular response rates during atrial fibrillation. The effects are modest, and at this point unlikely to impact clinical care. ${ }^{[25]}$

\section{Advance Genetic and Genomic Technologies \\ Many of the causative genes described were identified using a candidate gene approach, in which genes are selected based on findings of preceding genetic linkage analysis or molecular pathway information. The human genome encodes at least 20,000 protein- coding genes, the candidate gene approach focuses only on a small fractions of genome with the reminder unanalyzed. Genome wide association studies (GAWS) using single nucleotide polymorphisms (SNPs) can significantly expendite linkage analysis by narrowing the regions of interest for further directed sequencing. GAWS has been used in the cardiac electrophysiological field and has resulted in the identification of several loci involved in long QT syndrome plays an important role in the calcium signaling pathways in myocardial repolarization, and many other ECG parameters.}

GAWS on heart rate revealed the genetic heterogeneity of heart regulation and 21 loci were identified including HCN4, gap junction gene GJA1, and the atrial $\alpha$-myosine heavy chain gene MYH6. The GWAS technique has been applied to identify multiple loci in which polymorphisms contribute to variability in the QT interval and other intervals on the electrocardiogram. The strongest QT signal is surprisingly near the NOS1AP gene, encoding an ancillary protein for neuronal nitric oxide synthase, and not previously implicated in cardiac electrophysiology ; one report implicates the encoded protein (termed CAPON) and as a modulator of electrical signaling in heart, but confirmatory data remain lacking. Interestingly, these GWAS analyses of the QT interval have also implicated common variation at the congenital long QT syndrome disease genes as a modulator of QT interval. That is, rare variants in these genes may cause the congenital long QT syndrome while common variants contribute to variability in the QT interval in the population. Interestingly, variants in QT GWAS loci (in $\mathrm{KCNH} 2$, NOS1AP and KCNQ1) have been implicated as modulators of the clinical severity of the congenital long QT syndromes, i.e. common variants appear to contribute to variable penetrance. This is an example of how gene-gene interactions may identify clinical subsets with extreme values of human traits such as arrhythmia susceptibility. ${ }^{[26]}$

\section{Bioelectronic medicine:}

Bioelectronic medicine are electronic devices can be used to analyse and modulate the electrical activity within nerves system.

Bioelectronic medicines are new and innovative approach of personalized drug delivery system. These are used to diagnosis of any disease, clinical testing and therapy. The main feature of bioelectronic medicine as it stimulate electrical impulse to nerves system and body tissues instead of chemical treatment.

Following are some bioelectronic devices are used for diagnosis and treatment of arrhythmia:

Implantable cardioverter defibrillator (ICD): It is battery powered device which placed under the skin and track heart rate. A battery powered pulse generator is implanted in a pouch under the skin of the chest or abdomen, just below the collarbone. Wires run from the pulse generator to position on the surface of or inside the heart and can be installed through blood vessels, which eliminate the need of open surgery.

It is used for treatment of ventricular arrhythmia, heart attack, sudden cardiac arrest, long QT syndrome, Burgada syndrome and congenital heart disease.

It acts as pacemaker and send electrical signal to heart when heart beat is too fast, it helps to stop abnormal rhythm by giving defibrillation shock.

Pacemaker: It is a small battery operated device that which helps to beat heart in regular rhythm. It consist of two parts, generator and wire. The generator produces electrical impulses that helps to stimulate heart beat. It is implanted under skin through small incision the generator is connected to the heart through tiny wires. Some pacemakers are external and temporary, not implanted surgically. 
The sinoatrial node is natural pacemaker of the heart. It produces the electrical impulses which are responsible for heart beat. For the heart to beat properly, the signal must travel down a specific path to reach the ventricles. In case of arrhythmic condition SA node becomes defective, the heartbeat may be too fast, too slow or irregular.

The pacemaker pulse generator sends electrical impulses to heart to help it pump properly. An electrode is placed next to the heart wall and small electrical charges travel through the wire of the heart.

Holter monitor diary: it is one kind of record of daily activities and symptoms during test. If person who is under treatment feeling symptoms such as chest pain, shortness of breath, dizziness should note in the holter monitor diary. This will compared with ECG of the person under treatment. Doctor needs a complete picture of daily activities and symptoms.

Wearable sweat sensor analyzer: it is small, wearable sensor that can read the molecular composition of sweat and send its signal to a smartphone. ${ }^{[27]}$

\section{Conclusion}

Personalize drug delivery system is most effective way to treat cardiac arrhythmia and arrhythmic syndrome safely. It helps to avoid different side effects of antiarrhythmic drug which are commonly used in the treatment of arrhythmia. It is customize for each person who is under treatment, it give treatment according to persons genetic makeup hence it also called as customize drug delivery system.

Now a days some devices also available in the market which helps to diagnose the disease are also mention in this review.

\section{References}

[1] Stratified, personalised or P4 medicine: a new direction for placing the patient at the centre of healthcare and health education (Technical report). Academy of Medical Sciences. May 2015. Retrieved 6 Jan 2016.

[2] "Many names for one concept or many concepts in one name?" . PHG Foundation. Retrieved 6 Jan 2015.

[3] "Personalized Medicine 101" . Personalized Medicine Coalition. Retrieved 26 April 2014.

[4] "What Is Arrhythmia?". National Heart, Lung, and Blood Institute. July 1, 2011. Archved from the original on 2 March 2015. Retrieved 7 March 2015.

[5] Camm AJ, Lip GY, De Caterina R et al. 2012 focused update of the ESC Guidelines for the management of atrial fibrillation: an update of the 2010 ESC Guidelines for the management of atrial fibrillation. Developed with the special contribution of the European Heart Rhythm Association. Eur. Heart J. 33(21), 2719-2747 (2012).

[6] Shen, H; Choe, W (April 2011). "Spontaneous highfrequency action potential". Science China. Life sciences. 54 (4): 311-35. doi:10.1007/s11427-0114157y . PMID 21509656.

[7] Schwartz PJ, Ackerman MJ, George AL Jr, Wilde AAM. Impact of Genetics on the Clinical Management of Channelopathies. Journal of the American College of Cardiology. 2013; 62:169- 180. [PubMed: 23684683].

[8] Cutler MJ, Kaufman ES. To Be or Not to Be: LongQT Syndrome Type 9. Circulation: Cardiovascular Genetics. 2013; 6:439-440. [PubMed: 24129590].

[9] Barrett ML, Smith MW, Elizhauser A, Honigman LS, Pines JM (December 2014). "Utilization of Intensive Care Services, 2011" . HCUP Statistical Brief \#185. Rockville, MD: Agency for Healthcare Research and Quality. Archived from the original on 2015-04-02.

[10] Roden DM, Tyndale RF. Genomic Medicine, Precision Medicine, Personalized Medicine: What/'s in a Name? Clin Pharmacol Ther. 2013; 94:169-172. [PubMed: 23872826].

[11] Food and Drug Administration. Hoechst Marion Roussel, Inc., and Baker Norton Pharmaceuticals, Inc., terfenadine; proposal to withdraw approval of two new drug applications and one abbreviated new drug application; opportunity for a hearing. Fed Regist 1997;62(9):1889-1892. April 8, 2017.

[12] Food and Drug Administration. Additions and modifications to the list of drugs that have been withdrawn or removed from the market for reasons of safety or effectiveness. Fed Regist 2014;79(127):37687- 37696.content/pkg/FR-2014-0702/pdf/201415371.pdf. Accessed April 8, 2017.

[13] Ioannidis JP, Ntzani EE, Trikalinos TA, ContopoulosIoannidis DG. Replication validity of genetic association studies. Nature Genetics. 2001; 29:306309.

[14] Lehnart SE, Ackerman MJ, Benson Jr DW, Inherited arrhythmias: a national heart, lung and blood institute and office of rare diseases workshop consensus report about the diagnosis, phenotyping, molecular mechanisms, and therapeutic approaches for primary cardiomyopathies of gene mutations affecting ion channel function, circulation 2007; 116; 2325-45.

[15] Ackerman MJ, Priori SG, Willems S, HRS/EHRA expert consensus statement on the stste of genetic testing for the channelpathies and cardiomyopathies. Heart rhythm 2011; 8:1308-39.

[16] Gollob MH, Blier I, Brugada R, Recommendations for the use of genetic testing in the clinical evaluation of inherited cardiac arrhythmias associated with sudden cardiac death: Canadian Cardiovascular society/ Canadian Heart Rhythm Society joint position paper, Can J Cardiol 2011; 27:232-45.

[17] Priori SG, Wilde AA, Horie M, HRS/EHRA/APHRS expert consensus statement on the diagnosis and management of patients with inherited primary arrhythmia syndromes. J Arrhythmia 2014 ; 30:29-47.

[18] Antzelevitch C, Yan G-X, Ackerman M-J, J-Wave syndromes expert consensus conference report: Emerging concepts and gaps in knowledge, J Arrhythmia 2016; 32(5):315-39. 